\title{
VITREOUS DETACHMENT AND THE POSTERIOR HYALOID MEMBRANE: A CLINICOPATHOLOGICAL STUDY
}

\author{
MARTIN P. SNEAD ${ }^{1}$, DAVID R.J. SNEAD ${ }^{2}$, ABDULAH S. MAHMOOD $^{2}$ and JOHN D. SCOTT ${ }^{1}$ \\ Cambridge and Bristol
}

\begin{abstract}
SUMMARY
This study examines the prevalence and clinicopathological features of posterior hyaloid membrane separation, or 'true' vitreous detachment, in a randomly selected population of general medical in-patients. One hundred and seven eyes of 60 patients were examined clinically using slit lamp biomicroscopy; in $57 \%$ the posterior hyaloid membrane was detached and in $43 \%$ the posterior hyaloid membrane was attached. Eighteen eyes have subsequently been examined immunohistochemically, demonstrating that the posterior hyaloid membrane, including the Weiss ring, is made up of type IV collagen. The implications of these findings are discussed.
\end{abstract}

The important relationship between vitreous pathology and retinal pathology at the vitreoretinal junction has been recognised for many years. ${ }^{1}$ More recently, interest has been focused particularly on the relationship between vitreous attachment or detachment and macular hole formation. ${ }^{2-4}$ The term 'vitreoschisis' has been introduced to describe fragmentation of the posterior vitreous gel without posterior hyaloid membrane separation ${ }^{5}$ or even splitting of the posterior hyaloid membrane itself. ${ }^{6}$

True vitreous detachment shows a distinct membrane on the posterior hyaloid surface and can be clearly observed clinically with slit lamp biomicroscopy as shown in Fig. 1 and in some instances induced or completed surgically. ${ }^{4,7,8}$ The posterior hyaloid membrane is tough, inelastic and has a crinkled surface. ${ }^{9}$ Where the gel is very collapsed the posterior hyaloid membrane can be observed directly with oblique slit lamp illumination alone (Fig. 1). A $90 \mathrm{D}$ condensing lens is required if the membrane is lying more posteriorly in the vitreous cavity.

The purpose of this study was to examine the prevalence and clinicopathological features of posterior hyaloid

From: 'Addenbrooke's Hospital, Cambridge; ${ }^{2}$ Bristol Royal Infirmary, Bristol, UK.

Correspondence to: M.P. Snead, FRCS, FRCOphth, Vitreoretinal Fellow, Clinic 3, Addenbrooke's Hospital, Hills Road, Cambridge CB2 2QQ, UK. membrane separation, or 'true' vitreous detachment, in a randomly selected population of general medical in-patients.

\section{METHODS}

\section{Part 1}

Prior ethical approval for the prospective study was granted and informed written consent was received from all patients recruited. A full past ocular and medical history was recorded together with details of present and past medication. The protocol excluded patients with a history of familial eye disease or previous intraocular surgery excluding uncomplicated extracapsular cataract extraction. All patients underwent an ocular examination comprising: visual acuity and colour vision assessment with Ishihara plates; anterior segment examination and applanation tonometry; dilation of pupils with cyclopentolate $1 \%$ and phenylephrine $10 \%$; biomicroscopic fundus examination in all patients with a slit lamp and $90 \mathrm{D}$ lens and indirect ophthalmoscopy with scleral depression. Where possible, corroborative photographs were also recorded.

Vitreous detachment was defined as a clearly visible continuous posterior hyaloid membrane. Eyes without media of sufficient clarity to make this judgement were not recruited to the study. A diagram was made of the particular configuration of a Weiss ring incorporated in the posterior hyaloid membrane to assist surgical identification during Part 2 of the study. In one instance it was possible to photograph the Weiss ring (Fig. 2).

\section{Part 2}

The hospital records of patients recruited to the study were flagged and, in the event of a patient's death, relatives were approached for informed consent for organ donation for research purposes. Where consent was received, the eyes were enucleated within 24 hours and fixed in $10 \%$ buffered formal saline prior to surgical, histological and immunohistochemical examination. 


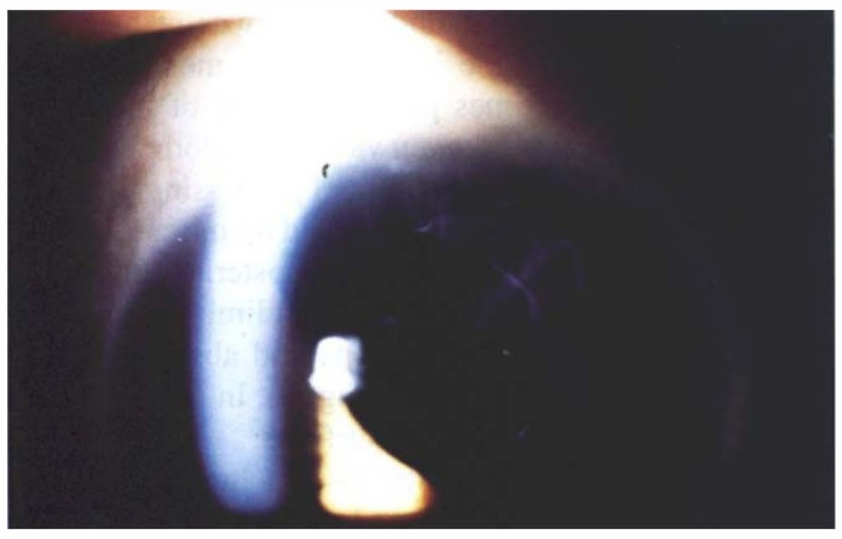

Fig. 1. Vitreous detachment. Very collapsed gel allows direct slit lamp view of the posterior hyaloid membrane. Note the characteristic wrinkled 'Cellophane-like' sheen.

\section{Pathology and Immunohistochemistry}

The eyes were fixed in $10 \%$ buffered formal saline for variable lengths of time up to 14 days. All eyes were then sliced into two calottes with a single vertical coronal incision lateral to the limbus. Both calottes were then placed into deep cassettes or metal baskets and processed into paraffin wax.

All specimens were processed on a standard Tissue Tek VIP automated vacuum processor (Miles Inc., Elkhart, USA). Three separate processing programmes were employed:

Programme 1: A standard 13.5 hour cycle consisting of 90 minutes in 10\% formal saline followed by 45 minutes each in graded alcohols $(70 \%, 80 \%), 60$ minutes in 95\% alcohol and then 4 hours in absolute alcohol. Specimens were then cleared in toluene for 2 hours with all steps at $40{ }^{\circ} \mathrm{C}$. This was followed by 3 hours in wax at $60^{\circ} \mathrm{C}$.

Programme 2: A 72 hour programme consisting of 60 minutes in $10 \%$ formal saline, 3 hours in 50\% alcohol, 4 hours each in $70 \%$ then $90 \%$ alcohol, 12 hours in absolute alcohol, 1 hour in 50:50 absolute alcohol and toluene followed by 24 hours in toluene and 14 hours in wax.

Programme 3: A 72 hour programme using chloroform as the clearing agent, consisting of 1 hour in $10 \%$ formal saline, 3 hours in 50\% alcohol, 4 hours each in $70 \%$ then $90 \%$ alcohol, 12 hours in absolute alcohol, then 1 hour in 50:50 alcohol and $100 \%$ chloroform, 14 hours in $100 \%$ chloroform and 14 hours in wax at $60{ }^{\circ} \mathrm{C}$.

Two eyes were noted clinically to have a particularly pronounced, thickened Weiss ring of specific configuration. The orientation and location of these rings had been documented diagrammatically, and in one case photographically, in Part 1 of the study. These eyes were dissected using the operating microscope, microsurgical instruments and vitrectomy so that the Weiss rings could be mobilised and removed alone. These were fixed to glass slides with histoacryl adhesive and air dried for direct immunohistochemical staining, together with fragments of the detached posterior hyaloid membrane, which were also removed. The specimens were stained with the type IV collagen stain as described below, omitting the deparaffinisation and trypsin steps. A plain slide with histoacryl glue was stained in the same manner as a control.

\section{Staining}

Sections were cut at $5 \mu \mathrm{m}$ thickness and stained with haematoxylin and eosin using a standard automated staining machine. Type IV collagen staining was performed using the monoclonal mouse anti-human type IV collagen antibody (Dakopatts A/S, Glostrup, Denmark) according to the manufacturer's guidelines using the streptavidinbiotin method. Paraffin sections were de-waxed in xylene, immersed in $10 \%$ hydrogen peroxide for 10 minutes to block endogenous binding, washed in phosphate-buffered saline (PBS), pre-digested with $1 \%$ trypsin for 10 minutes and washed in water. The primary antibody diluted with PBS and rabbit serum $(100 \mu \mathrm{l} / 10 \mathrm{ml})$ was applied for 60 minutes. After rinsing with PBS, the biotinylated rabbit anti-mouse antibody was applied for 30 minutes, then washed in PBS and the horseradish peroxidase (HRP) conjugated streptavidin-biotin applied for 30 minutes and then washed in PBS. The DAB working solution was applied for 20 minutes, washed in water, and counterstained with Mayer's haematoxylin. Negative controls were carried out using the same method but excluding the application of the primary antibody.

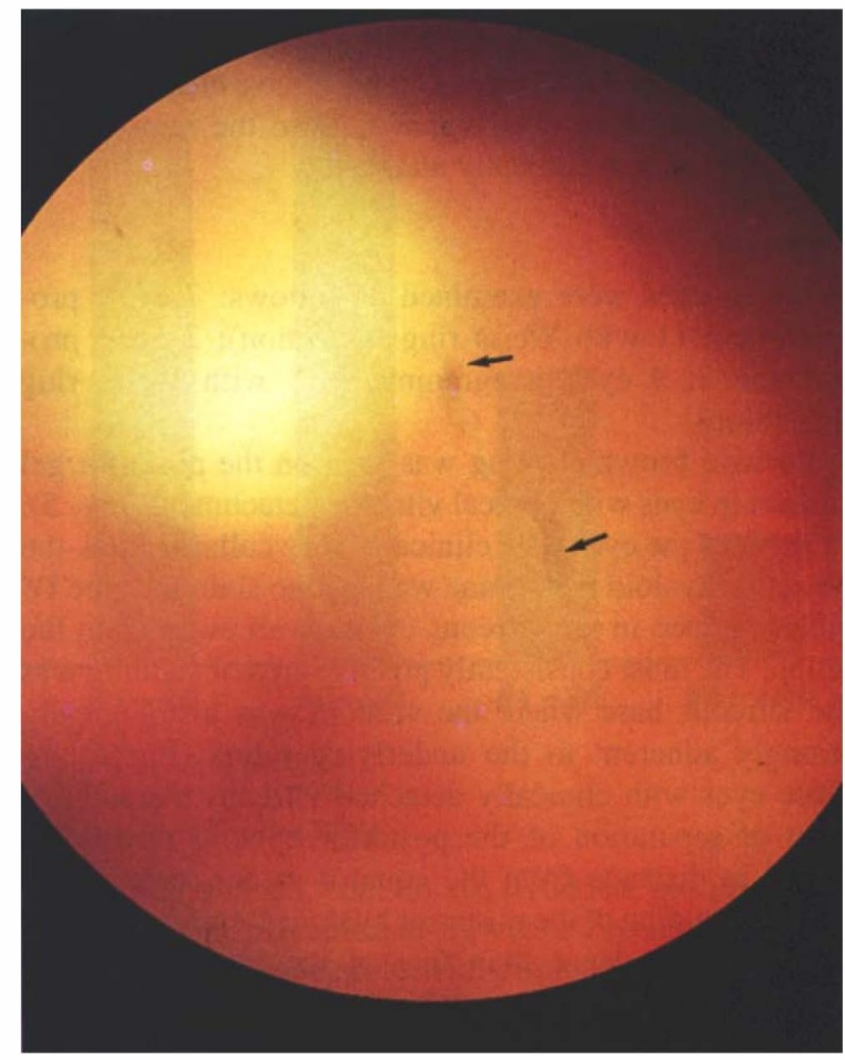

Fig. 2. Fundus photograph of a 68-year-old woman with posterior hyaloid membrane detachment incorporating Weiss ring with distinctive thickened lateral border (arrows). 


\section{RESULTS}

Sixty patients (120 eyes) were examined. Thirteen eyes had media opacity or had had previous posterior segment surgery and were excluded, so that 107 eyes remained in the study. The following factors were considered unlikely to compromise the project and were therefore included:

1. Age-related macular degeneration of varying degrees (23 eyes).

2. Uncomplicated extracapsular cataract surgery without YAG laser capsulotomy (2 eyes).

3 . Asymptomatic flat retinal tears ( 2 eyes).

4. Flat naevus ( 1 eye).

5. Asymptomatic macroaneurysm (1 eye).

Fifteen men and 45 women were examined. Their ages ranged from 68 to 98 years with a mean age of 83.4 years. There was no significant difference $(p>0.05)$ in the age ranges for eyes with attached and detached posterior hyaloid membranes. Fourteen patients have died during the 18 month study and in 9 (18 eyes) informed consent was given for surgical and histological examination of the posterior hyaloid membranes (Part 2). In 5 consent was not given and these eyes were therefore not included in Part 2.

\section{Vitreous Detachment}

In 61 eyes $(57 \%)$ the posterior hyaloid membrane had clearly separated from the retina and in 46 eyes (43\%) the vitreous remained attached. Figs. 3 and 4 illustrate the age distributions for eyes with attached and detached posterior hyaloid membranes. Of the 18 eyes subsequently examined histologically, 11 were known to have clear posterior hyaloid membrane detachments and in the remaining 7 eyes, the gel was clinically attached.

\section{Histology}

Eighteen eyes were examined as follows: 7 eyes, programme 1 (1 with Weiss ring dissection); 2 eyes, programme 2; 9 eyes, programme 3 ( 1 with Weiss ring dissection).

Positive brown staining was seen on the posterior gel surface in eyes with clinical vitreous detachment (Fig. 5). In some of the eyes with clinically very collapsed gel, the posterior hyaloid membrane was seen to stain for type IV collagen deep in the vitreous cavity, well away from the retina. The most consistently positive area of staining was the vitreous base where the vitreous was histologically strongly adherent to the underlying retina (Fig. 6). In those eyes with clinically detached vitreous the anterior limit of separation of the posterior hyaloid membrane varied in distance from the equator to ora serrata. The positive staining of the posterior hyaloid membrane was in all cases less dense than that of the internal control, namely, the basement membrane of the retinal blood vessels (see Fig. 7).

Staining of the detached posterior hyaloid membrane was most effective using the long 72 hour cycle with chloroform as the clearing agent (programme 3 ). In eyes pro- cessed by programmes 1 and 2 there was at best only equivocal staining of the inner limiting membrane and only light and sometimes patchy staining of the retinal blood vessels. In one eye, even vascular endothelial staining was equivocal with programme 1 processing. In all but one eye with clinical posterior hyaloid membrane detachment the staining was confined to the posterior gel, joining the vitreoretinal interface at the anterior limit of separation of the posterior hyaloid membrane and absent from the retinal surface posterior to this (Fig. 7). In the remaining eye there was staining on both surfaces.

In the eyes with clinical vitreous attachment, the staining was confined to the interface between retina and overlying adherent vitreous (Fig. 8a,b).

The independently stained Weiss rings, although slightly disrupted during staining, were strongly positive for type IV collagen as were fragments of adjoining posterior hyaloid membrane (Fig. 9). The negative control histoacryl glue did not take up stain.

\section{DISCUSSION}

This is the first prospective clinicopathological study of the detached posterior hyaloid membrane in a large series of patients. The results compare with $75.4 \%$ with vitreous attachment and $24.5 \%$ with vitreous detachment in the 786 subjects aged $20-80+$ years examined solely post mortem by Foos. ${ }^{10}$

Positive evidence of a Weiss ring incorporated within the separated posterior hyaloid membrane can be used as a useful tool to aid histological identification of the membrane and its structure. In the clinical setting, however, identification of the ring is not always possible or necessary as it can be disrupted, for example, by a large gel herniation through the membrane into the preretinal space or by differences in separation from the disc and peripapillary retina.

The most effective method of processing was found to be a long 72 hour cycle using chloroform as the clearing agent. This allows optimum morphology by decreasing the brittleness of the tissue ${ }^{11}$ and facilitates section cutting. It also appeared to give improved sensitivity to the type IV collagen stain. The reasons for this are unclear but it is possible that the prolonged processing time allows superior unmasking of the antigen, in which case a long predigestion time may also improve sensitivity.

Type IV collagen, along with laminin, acts as the scaffold for basement membrane formation and is a unique intrinsic structural component of basement membranes. ${ }^{12.13}$ This study clearly indicates that the detached posterior hyaloid membrane including a Weiss ring is composed of type IV collagen and represents delamination of the inner limiting membrane of the retina. This endorses the clinicopathological case report of Funata $e t$ $a l .{ }^{14}$ who demonstrated large areas of inner limiting membrane defect following posterior hyaloid membrane surgical stripping in 2 eyes with attached vitreous prior to surgery. Type IV collagen in the region of the inner limiting membrane has been identified previously by others ${ }^{15}$ 


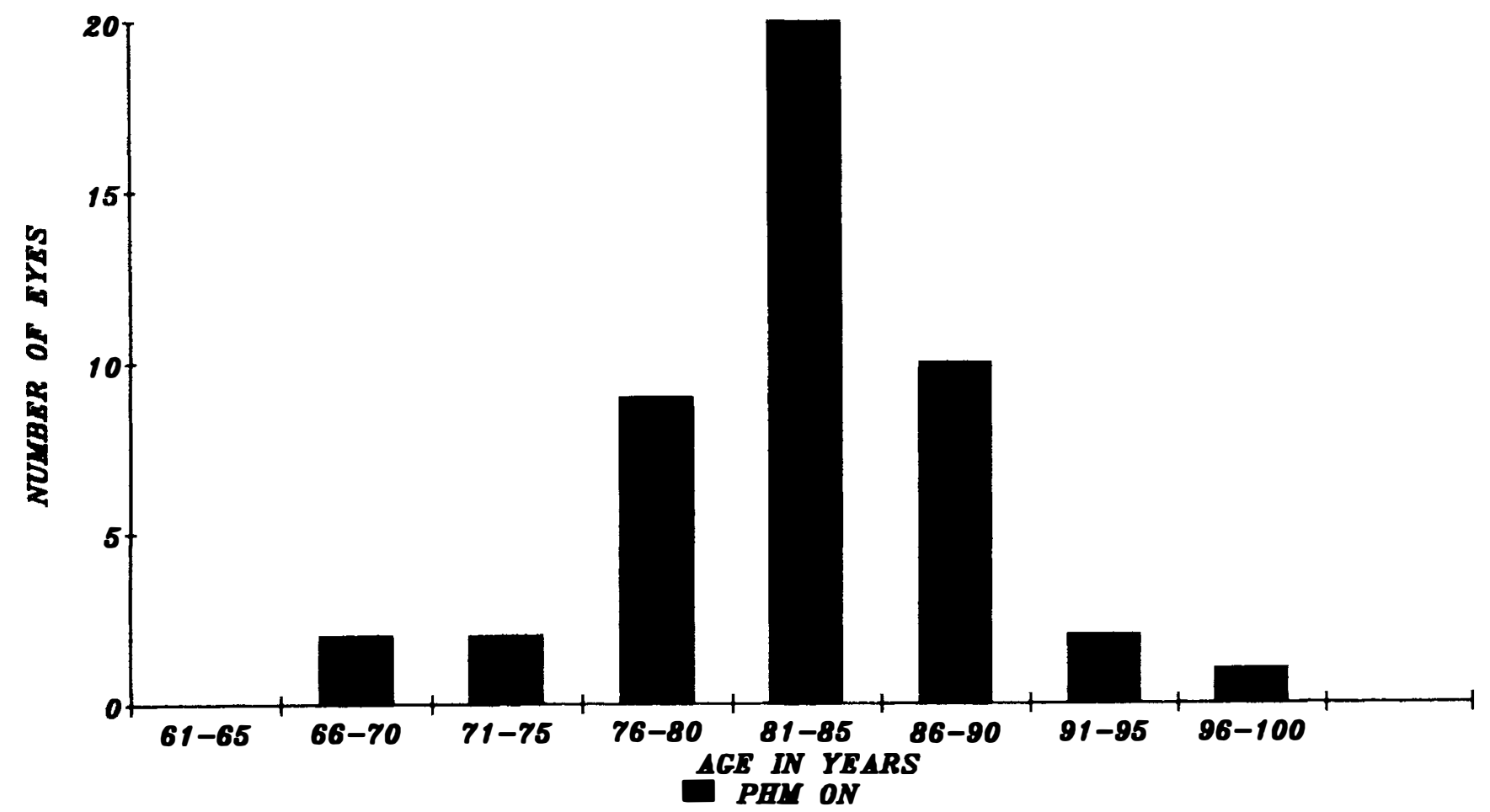

Fig. 3. Age range and distribution for eyes with attached posterior hyaloid membrane (PHM).

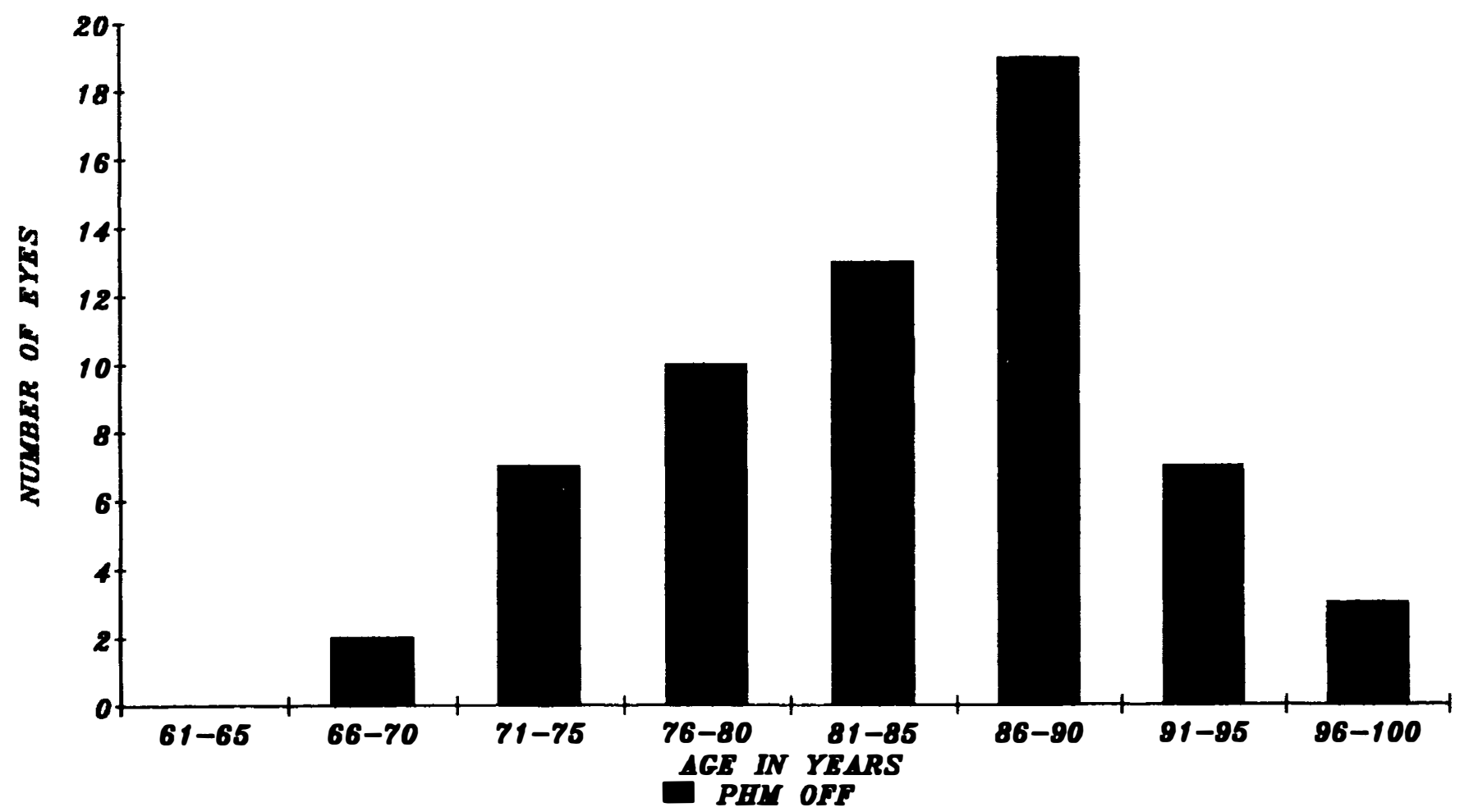

Fig. 4. Age range and distribution for eyes with detached posterior hyaloid membrame (PHM).

and the presence of basement membrane at the posterior vitreous border has been suggested by Gartner. ${ }^{16}$ Type IV collagen presumed to represent areas of inner limiting membrane in surgically segmented proliferative vitreoretinopathy membranes has been identified by many workers. $^{17-20}$

It is important to emphasise that this study defines clinical (ante-mortem) vitreous detachment solely on the basis of a clearly defined, continuous, wrinkled, posterior hyaloid membrane. Myopic and age-related syneretic vitreous change is still compatible with clinical and histological 'true' vitreous attachment (Fig. 8a). The relationship of the inner limiting membrane and its separation or otherwise from the surface of the posterior retina has important implications for both the pathogenesis ${ }^{3}$ and the treatment of macular (and impending macular) holes. ${ }^{4,14,18}$ The 


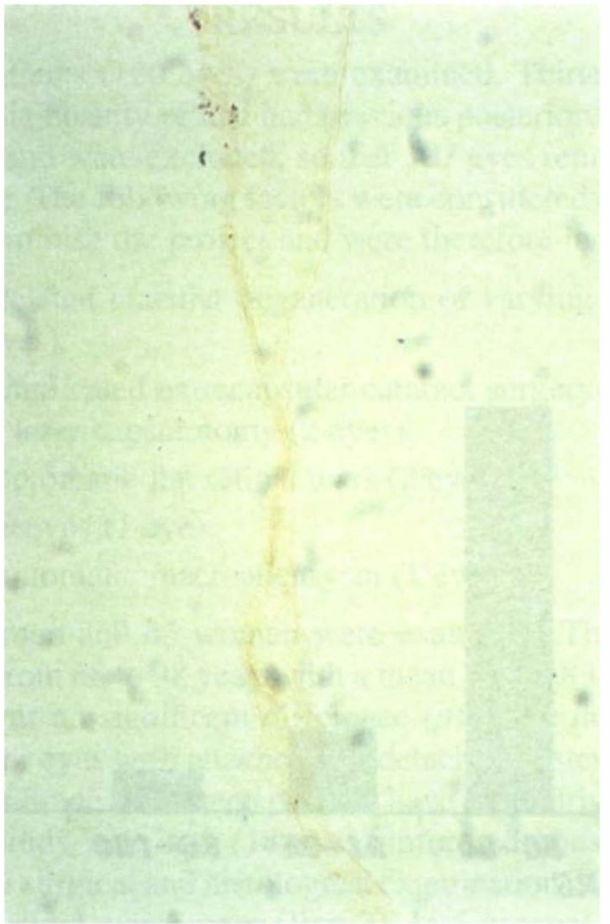

Fig. 5. Vitreous detachment. Positive brown stain over the posterior gel in an 89-year-old man with clinically detached posterior hyaloid.membrane $(\times 250)$. Acuity $6 / 12$ with -2.00 DS, cryopexy to flat horseshoe tear 1973.

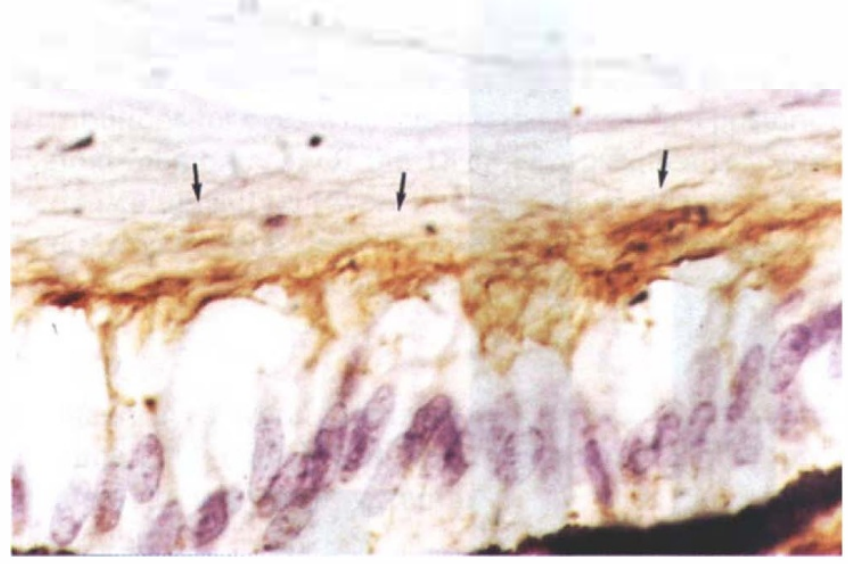

Fig. 6. Same patient as in Fig. 5. Dense type IV collagen stain is seen (brown, arrows) at the vitreous base $(\times 400)$.

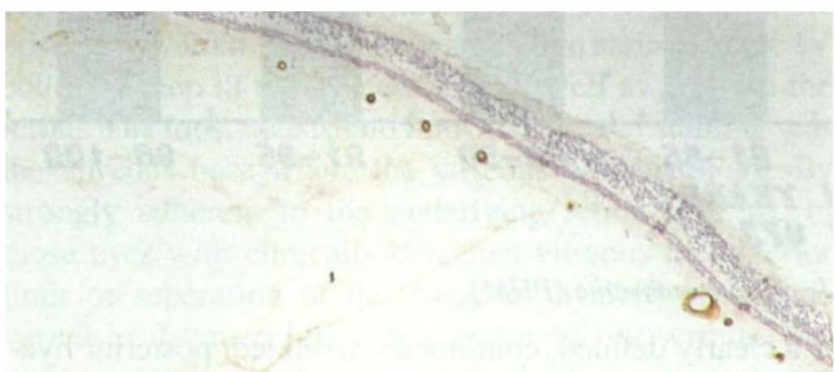

Fig. 7. An 81-year-old man with clinically detached posterior hyaloid membrane showing the anterior limit of membrane separation between ora serrata and equator. Type IV collagen stain ( $\times 100)$. See text for details.

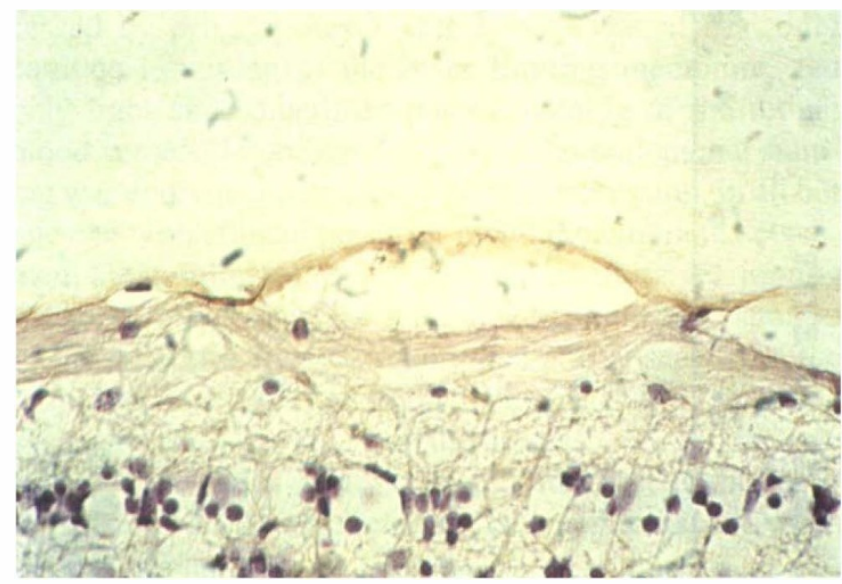

Fig. 8a. An 81-year-old woman (a low myope since age 20 years) with vitreous syneresis but clinically attached posterior hyaloid membrane. Corrected visual acuity 6/9 right and left.

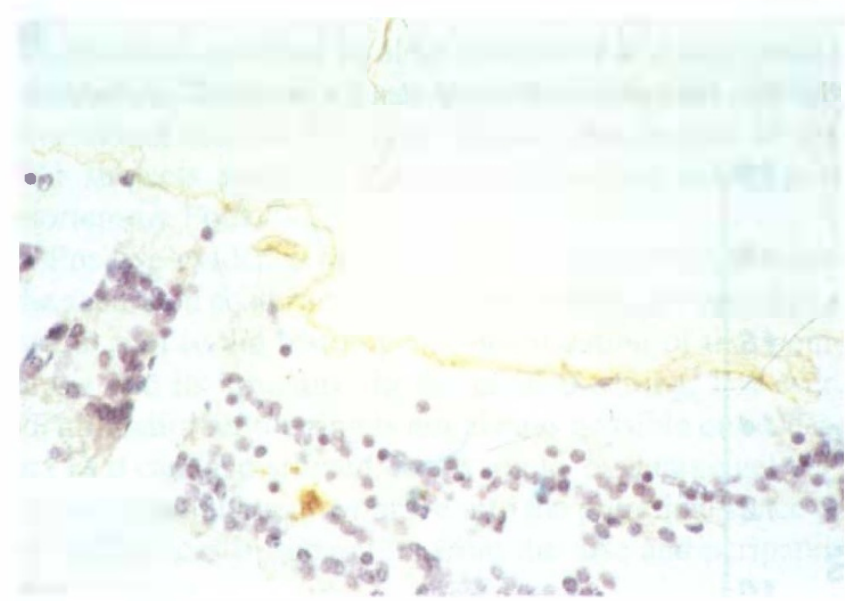

Fig. 8b. A 70-year-old woman with clinically attached gel. Corrected visual acuity 6-6 right and left. Note: Type IV collagen stain is confined to the vitreoretinal interface $(\times 250)$.

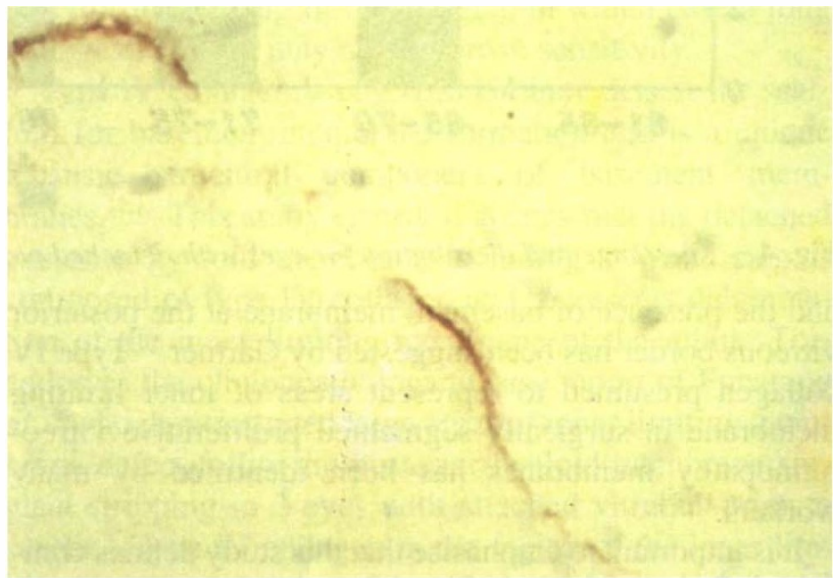

Fig. 9. Weiss ring from a 68-year-old woman (same eve as in Fig. 2). The thickened border stains strongly positively for type IV collagen $(\times 400)$. 
results of this study also indicate that, in eyes with vitreous detachment, the concept of inner limiting membrane preservation and sanctity during vitrectomy in order to reduce the risk of proliferative vitreoretinopathy ${ }^{21}$ may need to be re-examined.

It is not clear from this study whether the inner limiting membrane can indeed split or even regenerate, and further clinicopathological studies are required in this area.

Key words: Posterior hyaloid membrane, Posterior vitreous detachment, Vitreoretinal junction, Vitreous disorders.

\section{REFERENCES}

1. Hruby K. The importance of the vitreous body. In: Schepens $C$, editor. Retina surgery with special emphasis on reoperations. Second conference of the Retina Foundation 1958. St Louis: CV Mosby, 1960.

2. Johnson RN, Gass JDM. Idiopathic macular holes: observations, stages of formation and implications for surgical intervention. Ophthalmology 1988;95:917-24.

3. Gass JDM. Idiopathic senile macular hole: its early stages and pathogenesis. Arch Ophthalmol 1988;106:629-39.

4. Kelly NE, Wendel RT. Vitreous surgery for idiopathic macular holes: results of a pilot study. Arch Ophthalmol 1991; 109:654-9.

5. Sebag J. Anatomy and pathology of the vitreo-retinal interface. Eye 1992;6:541-52.

6. McLeod D. Editorial. Eye 1992:6:vii-viii.

7. Snead MP. Current ocular therapy with silicone oils. Curr Opin Ophthalmol 1993;4:36-43.

8. Vander JF, Kleiner R. A method for induction of posterior vitreous detachment during vitrectomy. Retina 1992;12: 172-3.

9. Scott JD. Prevention and perspective in retinal detachment. Eye 1989;3:491-515.

10. Foos RY. Posterior vitreous detachment. Trans Am Acad Ophthalmol Otolaryngol 1972;76:480-97.
11. Gordon KC. In: Bancroft JD, Stevens A, editors. The theory and practice of histological techniques, 2 nd ed. Edinburgh: Churchill Livingstone, 1982:41-60.

12. Yurchenco PD. Assembly of basement membranes. Ann NY Acad Sci 1990:580: 195-213.

13. Kuhn K, Glanville RW, Babel W, Quin R-Q, Dieringer H, Voss T, et al. The structure of type IV collagen. Ann NY Acad Sci 1985;460:14-24

14. Funata M, Wendel RT, De La Cruz Z, Green WR. Clinicopathologic study of bilateral macular holes treated with pars plana vitrectomy and gas tamponade. Retina 1992;12: 289-98.

15. Jerdan JA, Pepose JS, Michels RG, Hayashi H, De Bustros S, Sebag M, Glaser BM. Proliferative vitreoretinopathy membranes: an immunohistochemical study. Ophthalmology 1989;96:801-10.

16. Gartner J. Histologische Beobachtungen über die Struktur der vitreretinalen Grenzschicht. Klin Monatsbl Augenheilk 1962;141:261.

17. Jerdan JA, Pepose JS, Michels RG, Hayashi H, De Bustros S, Sebag M, Glaser BM. Proliferative vitreoretinopathy membranes: an immunohistochemical study. Ophthalmology 1989:96:801-10.

18. Green WR, Kenyon KR, Michels RG, et al. Ultrastructure of epiretinal membranes causing macular pucker after retinal reattachment surgery. Trans Ophthalmol Soc UK 1979;99: 63-77.

19. Cleary PE, Minckler DS, Ryan SJ. Ultrastructure of traction retinal detachment in rhesus monkey eyes after a posterior penetrating ocular injury. Am J Ophthalmol 1980;90: $829-45$.

20. Kampik K, Green WR, Michels RG, Nase PK. Ultrastructural features of progressive idiopathic epiretinal membrane removed by vitreous surgery. Am J Ophthalmol 1980;90: 797-809.

21. Lais S, Rathschuller F, Colacino R, Ciurlo G. Alterations de la lame limitante interne de la retine après pelage des membranes epiretiniennes. J Fr Ophtalmol 1991;14:647-9. 